\title{
Explaining variation in energy balance using high density SNP information
}

\section{K.L. Verbyla ${ }^{1,2}$, M.P.L. Calus ${ }^{1}$, Y. de $\operatorname{Haas}^{1}$, H.A. Mulder ${ }^{1}$, R.F. Veerkamp ${ }^{1}$}

${ }^{1}$ Animal Breeding and Genomics Centre,Wageningen UR Livestock Research, Lelystad, Netherlands, ${ }^{2}$ The University of Melbourne, Victoria, Australia, ${ }^{3}$ The Department of Primary Industries, Victoria, Australia, ${ }^{4}$ The Cooperative Research Centre for Beef Genetic Technologies, Armidale, Australia

Email: k.verbyla@pgrad.unimelb.edu.au

Introduction Severe negative energy balance (EB) has been shown to have harmful effects on health and fertility. Estimates of heritability and genetic correlations suggest that EB is not only a consequence of a poor match between nutrition and production, but is also genetically induced (Veerkamp et al.; 2003, Friggens et al., 2007). The importance of EB to animal breeders comes from the high costs of involuntary culling caused by poor health or failing to establish a successful pregnancy caused by impaired ovarian function or delayed resumption of oestrous cycles. Currently, high density SNP chips are available to investigate the genetics of EB and the use of genomic selection could increase the ability to select for complex traits such as EB. The aim of this study was to demonstrate that high density SNP information can be used to estimate genomic breeding values (GEBV) for genetically induced EB.

Material and methods A total of 588 Holstein-Friesian heifers with known pedigree born between 1990 and 1997 were genotyped for the Illumina 50K SNP panel (54,001 SNPs in total). Quality control and pedigree checking was performed and 43,011 SNPs and 548 animals were retained. During the first 15 wk of lactation live weight, feed intake, and milk yield were measured on 527 of these heifers. All heifers were fed ad libitum. Fat, protein, and lactose yields were measured at a fixed day of the week. Feed intake was recorded daily using automated feed intake units. EB (MJ/d) was calculated as the difference between energy intake and calculated energy requirements for milk, fat, and protein yields and maintenance costs as a function of live weight. The EB values were averaged across 14 weeks (2-15) to provide the EB phenotype that was used. A 10-fold cross validation approach was carried out, such that the data was randomly partitioned into 10 subsets. Each subset was retained once as the validation dataset and the remaining 9 sets were used to predict the GEBV of those animals in the validation set. The model described in Calus et al., (2008) was used to predict the GEBV. The GEBV were calculated as the sum of the estimated SNP effects and the polygenic effect. The same data subsets and approach were used with a simple polygenic model excluding the SNP information for comparison. The GEBV were assessed using accuracy $r_{y g}$ of the predicted GEBV $(g)$ when compared with the phenotypes $(y)$ and thus the $r_{y g}^{2}$. The accuracy of selection $\left(r_{g g}\right)$ when comparing the true breeding values $(\mathrm{g})$ and GEBV has been reported to be a function of the heritability, the number of phenotypic records and the number of effective QTL (Daetwyler et al., 2008 ; Goddard, 2009). This function was adapted for use with the accuracy when comparing phenotypes and GEBV. It was then applied to predict the number of effective QTL for EB and the number of records needed to reach different levels of accuracy of selection.

Results The model including the SNP information yielded an overall accuracy of 0.294 and thus an $r_{y g}^{2}$ of 0.086 $\left(r_{g g}^{2}=0.265\right)$ when comparing the phenotypes and GEBV in the combined validation sets. The maximum $r_{y g}^{2}$ that this model could have gained was equal to the heritability which was calculated separately as 0.325 . For the model excluding the SNP information with only the polygenic effect an overall accuracy of 0.211 and $r_{y g}^{2}$ of 0.044 was found. The effective number of QTL for EB was predicted to be 472 and a total of 5818 records with phenotype and genotype information was predicted as needed for an $\mathrm{r}_{\mathrm{gg}}^{2}$ of 0.80 .

Conclusions. The use of SNP information confirm the genetic background of EB. Using the SNP information an increase in the accuracy of selection for EB was achieved over the simple polygenic model. Thus, EB could be selected for using genomic selection. The size of the data set directly impacts the expected accuracy and thus an increase in the total number of phenotypic records used would be required to improve the accuracy of selection.

Acknowledgements The authors acknowledge funding from SABRETRAIN, Productschap Zuivel, Senter Novem and RobustMilk.

\section{References}

Calus, M.P.L., Meuwissen, T.H.E., De Roos, A.P.W., and Veerkamp, R.F. 2008. Genetics 178, 553-561.

Daetwyler, H.D., Villanueva, B., and Woolliams J.A. 2008. PLoS ONE. 3, e3395

Goddard, M.E. 2009. Genetica 136, 245-257

Friggens, N.C., Berg, P., Theilgaard, P., Korsgaard, I.R., Ingvartsen, K.L., Løvendahl, P. and Jensen, J. 2007. Journal of Dairy Science 90, 5291-5305

Veerkamp. R.F. Beerda, B., van der Lende., T. 2003. Livestock Production Science 83, 257-275 